\title{
On the removal of the open earcanal high-pass filter effect due to its occlusion: A bone-conduction occlusion effect theory
}

\author{
Kévin Carillo ${ }^{1, *}$ (D), Olivier Doutres ${ }^{1}$, and Franck Sgard ${ }^{2}$ \\ ${ }^{1}$ École de technologie supérieure, Montréal, Québec, Canada \\ ${ }^{2}$ IRSST, Montréal, Québec, Canada
}

Received 2 March 2021, Accepted 29 July 2021

\begin{abstract}
The occlusion effect is commonly experienced by in-ear device wearers as an increased loudness sensation of bone-conducted low frequency sounds. A widespread theory proposed by Tonndorf and based on a simplified electro-acoustic model describes the phenomenon as the removal of the open earcanal high-pass filter effect due to a perfect or partial occlusion. However, this filter has not been clearly defined and several ambiguities remain. Revisiting the model, a second order high-pass filter effect for the volume velocity transferred between the earcanal wall and the eardrum is highlighted. This filter remains for partial occlusion but vanishes for perfect occlusion. In the latter case, the volume velocity transferred from the earcanal cavity to the middle ear through the eardrum drastically increases, which explains the predominance of the occluded outer ear pathway on the hearing by bone-conduction at low frequencies.
\end{abstract}

Keywords: Occlusion effect, Bone conduction, Outer ear, High-pass filter effect

\section{Introduction}

The occlusion effect $(\mathrm{OE})$ is experienced as an increase of the auditory perception to bone-conducted sound when covering or blocking the earcanal (EC) [1]. In everyday life, the $\mathrm{OE}$ is usually described as one's own voice perceived as "hollow" or "talking into a barrel" [2]. This perception is the conjunction of the bone-conducted transmission increase and the air-conducted transmission decrease due to the occlusion device [3]. A common objective measurement of the $\mathrm{OE}$ is defined as the difference between sound pressure levels measured close to the tympanic membrane (TM) in occluded and open EC. This phenomenon is most prominent at low frequencies (LF), typically below $1 \mathrm{kHz}$, and for shallow occlusions [1].

Since the first descriptions of experimental findings underlying the OE in the 1830s, several theories were developed to explain this phenomenon over the course of the next century. Historical reviews of these theories can be found in $[4,5]$. In the 1960s, two explanations of the OE were developed. The first one, submitted by Huizing [4], was based on the change in resonance properties of the EC due to its occlusion. A few years later, this change was investigated experimentally by Tonndorf [5] and deemed valid only above $1 \mathrm{kHz}$. At LF, Tonndorf rather suggested that the $\mathrm{OE}$ resulted from the removal of the open EC high-pass filter effect due to the occlusion [5-7].

*Corresponding author: kevin.carillo.1@ens.etsmtl.ca
This theory relied on a simplified electro-acoustic (EA) model of the open and occluded EC. This model, involving acoustic mass and compliances, was the first model of the $\mathrm{OE}$ and has inspired several authors to date $[3,8]$. However, the open EC high-pass filter mentioned by Tonndorf has not been clearly defined and the simplified EA model has only been put forward to illustrate the concept, not to investigate the filter's parameters nor its removal due to a partial or perfect occlusion.

In the literature, the open EC filter effect has been mainly interpreted in terms of acoustic energy transfer [3, 9-12]. Tonndorf himself related the open EC filter effect to the acoustic energy dissipated through the open EC entrance: "The open EC acts as a high-pass filter so that low frequency sounds are dissipated through its external opening" [7]. However, since (i) acoustic resistances are not necessary to model the OE at LF [8] and (ii) Tonndorf's model itself did not include them to account for dissipation of acoustic energy, the filter effect of the open EC is not necessarily related to dissipation phenomena. Furthermore, the acoustic power dissipated through the EC opening is negligible at LF in the open case compared to the acoustic power dissipated in the EC cavity and at the TM, and its reduction due to the occlusion cannot explain the OE [8]. In the occluded case, Tonndorf explained that "the high-pass filter effect is eliminated, either partially or totally, depending upon the quality of the seal" [7].

Tonndorf's theory has been widely quoted in the literature to explain the fundamentals of the $\mathrm{OE}$ at LF until 
recently (e.g., [3, 9-14]). This theory, however, suffers from several ambiguities. To the authors' knowledge, no work has revisited in detail this widespread theory. The aim of the present paper is thus to investigate and clarify the open EC high-pass filter effect and how perfect and partial occlusions affect it. For this purpose, a revisited EA model inspired from Tonndorf's is presented. This work is believed to contribute to a better understanding of Tonndorf's theory, of the hearing by bone-conduction and of the fundamental mechanism of the $\mathrm{OE}$ at LF.

\section{Models of the occlusion effect \\ 2.1 Review of Tonndorf's model}

Figure 1 presents the EA model of an open and occluded EC proposed by Tonndorf [5] for a bone-conducted stimulation at LF. The source responsible for the acoustic pressure $\tilde{p}$ generated in the EC cavity is identified by Tonndorf to be the vibration of the $\mathrm{EC}$ wall. At that time, the mandibular condyle of the lower jaw was also believed to be a part of this source, but it has since then been shown to have little influence [14]. In the open case (see Fig. 1a), the air within the EC cavity is accounted for using an acoustic compliance $C_{\mathrm{EC}}$ near the TM and an acoustic mass $L_{\mathrm{EC}}$ near the EC opening. In the occluded case (see Fig. 1b), the EC cavity is approximated by an acoustic compliance $C_{\mathrm{EC}}$. In both cases, the TM is approximated by an acoustic compliance $C_{\mathrm{TM}}$.

The ambiguous points in Tonndorf's model are now highlighted. Firstly, the acoustic pressure generated in the EC cavity is identically referred to as $\tilde{p}$ in both open and occluded cases. It is certain that the acoustic pressure cannot be equal in both cases, or else the OE would be zero. In EA models subsequent to that of Tonndorf, the vibration of the $\mathrm{EC}$ wall has been represented as an equivalent and ideal source of volume velocity $[3,8,13,15]$ which is not affected by the acoustic load. The acoustic pressure generated by the source then depends on the EC acoustic properties which differ when the EC is open or occluded. Secondly, the separation between the portion of air controlled by the acoustic mass $L_{\mathrm{EC}}$ and that controlled by the acoustic compliance $C_{\mathrm{EC}}$ in the open case has not been detailed. In a previous paper, the authors showed that the junction between both portions is defined by the location of the equivalent volume velocity source in the EC [8]. In addition, the authors highlighted that the location of this source is not arbitrary but rather corresponds to the centroid position of the spatially distributed EC wall normal velocity. In the occluded case, the acoustic compliance $C_{\mathrm{EC}}$ only depends on the occluded EC residual volume. $C_{\mathrm{EC}}$ is thus not necessarily the same in both open and occluded cases, depending on the occlusion device insertion depth. Thirdly, the two compliances $C_{\mathrm{TM}}$ and $C_{\mathrm{EC}}$ in series in Tonndorf's model should be arranged in parallel since they share the same acoustic pressure [8], not the same volume velocity. Fourthly, the transfer function of the open EC, described by Tonndorf as a high-pass filter due to the acoustic mass $L_{\mathrm{EC}}$, has not been explicitly defined (i.e., physical nature of the input and output signals and order of the filter). In addition, this filter has been related to the EC opening acoustic "dissipation" while no acoustic resistance was accounted for in Tonndorf's model. Furthermore, the "removal" or "elimination" of this filter due to a partial or perfect occlusion of the EC opening has not been explicitly reported. Thus, Tonndorf's explanation of the OE remains unclear.

Since the first three ambiguous points have been clarified previously, we now focus on the fourth point related to the filter effect. As mentioned in Section 1, this clarification is important in the authors' opinion since the "removal of the open EC filter effect" is commonly used to explain the $\mathrm{OE}$ induced by perfect or partial occlusion.

\subsection{Revisited electro-acoustic model}

\subsubsection{Layout}

In order to investigate the open EC filter effect mentioned by Tonndorf and its change due to a perfect and a partial occlusion, three configurations are modeled here using a revisited EA model illustrated in Figure 2: (a) an open EC, (b) a perfectly occluded EC and (c) a partially occluded EC with a coaxial hole of radius $r_{\mathrm{h}}=0.9 \mathrm{~mm}$ and length $l_{\mathrm{h}}=21 \mathrm{~mm}$ (dimensions equivalent to those used by Hansen [16] for comparison with experimental data in Sect. 3). The EC is considered as a circular cross-sectional duct of curvilinear length $l_{\mathrm{EC}}=34.7 \mathrm{~mm}$ and varying radius $r_{\mathrm{EC}}(z)$, with $z$ the EC curvilinear axis. The EC shape is taken from a realistic 3D finite element (FE) model of an outer ear [8] and displayed in Figure 3. Perfect and partial occlusions are defined at the EC entrance and correspond to an extreme case of shallow insertion. The volume velocity $q_{\text {wall }}$ imposed by the EC wall represented by the source $Q$ is thus equal in all cases. The source $Q$ is located at a distance $l_{\mathrm{c}}=23.6 \mathrm{~mm}$ from the TM. This distance corresponds to the centroid position of the EC wall normal vibration and has been computed using the $3 \mathrm{D}$ FE model to which the current EA model is associated [8].

The source $Q$ divides the EC into an upstream section and a downstream section (see Fig. 2). In all cases, the downstream section is dominated at LF by its compressibility effect of acoustic compliance $C_{\mathrm{d}}$. The downstream section is terminated by the TM. The acoustic impedance $\hat{Z}_{\mathrm{TM}}$ (complex-valued) of the TM is defined by Shaw and Stinson's model [17]. In the open case, the upstream section is dominated by its inertia effect represented by the acoustic mass $L_{\mathrm{u}}$. The radiation impedance of the EC opening is modeled by the acoustic impedance of a baffled circular piston [18] (of radius $r_{\text {ent }}=2.8 \mathrm{~mm}$ equal to that of the EC entrance) and is governed at LF by an acoustic mass $L_{\text {rad. }}$. In the occluded case, the upstream section is dominated by the acoustic compliance $C_{\mathrm{u}}$. The expressions of localized constants $C_{\mathrm{d}}, C_{\mathrm{u}}, L_{\mathrm{u}}$ and $L_{\mathrm{rad}}$ derived from the 3D FE model [8] are provided in Table 1.

In the partially occluded case, the upstream section includes both the acoustic mass $L_{\mathrm{u}}$ and the acoustic compliance $C_{\mathrm{u}}$ since their respective importance depends on the hole impedance seen by the upstream section. The acoustic 


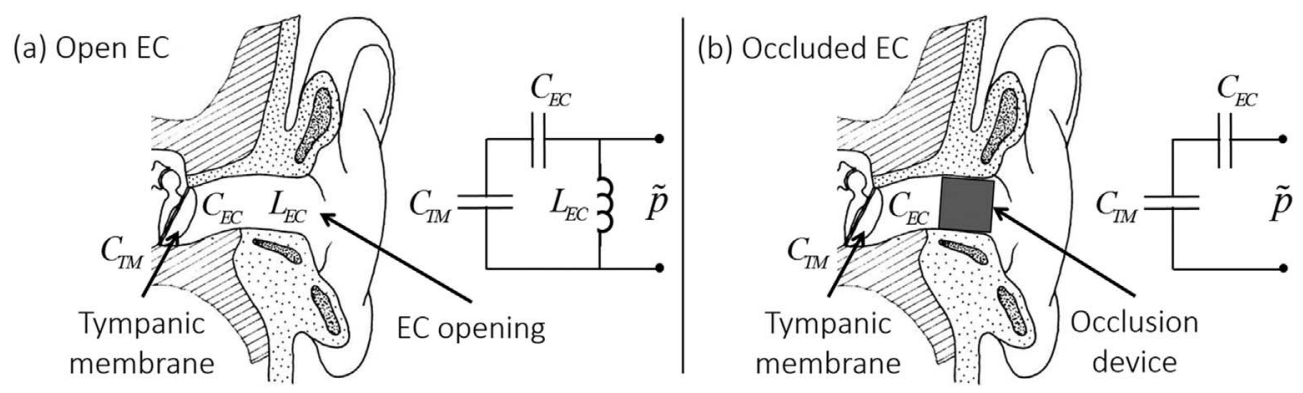

Figure 1. Tonndorf's EA model of (a) an open and (b) occluded EC (adapted from [5]).

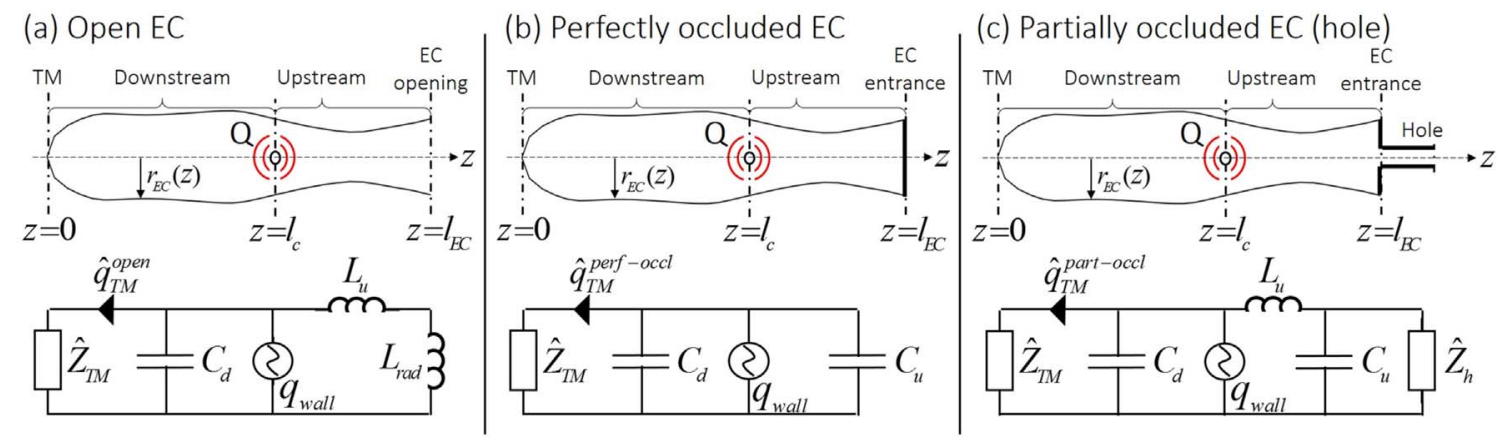

Figure 2. Revisited EA model of an (a) open, (b) perfectly occluded and (c) partially occluded EC.

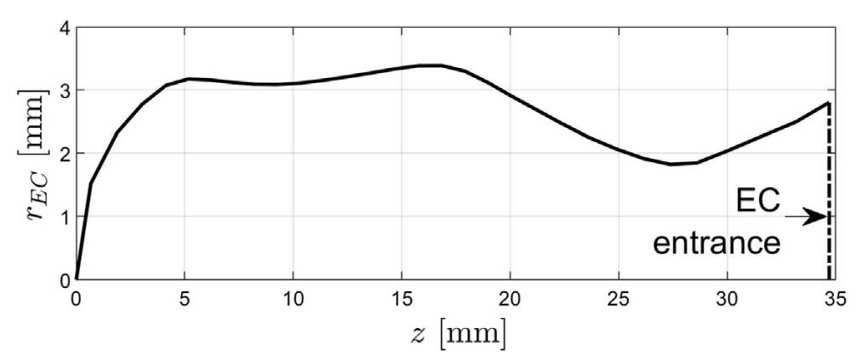

Figure 3. EC radius $r_{\mathrm{EC}}(z)$ as a function of the EC curvilinear axis $z$ used in the EA model.

impedance of the hole is defined by $\hat{Z}_{\mathrm{h}}=R_{\mathrm{h}}+j \omega L_{\mathrm{h}}^{\mathrm{eq}}$. The equivalent acoustic mass of the hole $L_{\mathrm{h}}^{\mathrm{eq}}=L_{\mathrm{h}}+L_{\mathrm{dis}}+L_{\mathrm{rad}}^{\mathrm{h}}$ includes the hole acoustic mass $L_{\mathrm{h}}$, the acoustic mass of discontinuity $L_{\text {dis }}$ due to the sudden change of section at the EC entrance [19], and the acoustic mass of radiation $L_{\text {rad }}^{\mathrm{h}}$ in the surrounding environment. The equivalent acoustic mass is associated with viscous losses represented by the acoustic resistance $R_{\mathrm{h}}$ [20]. Table 1 summarizes the calculation of $R_{\mathrm{h}}, L_{\mathrm{h}}, L_{\mathrm{dis}}$ and $L_{\mathrm{rad}}^{\mathrm{h}}$. It is noteworthy that the expressions of $R_{\mathrm{h}}$ and $L_{\mathrm{h}}$ are adapted for a "large" hole such that $\left|\hat{k}_{\mathrm{vi} / \mathrm{th}} r_{\mathrm{h}}\right|>10$ where $\hat{k}_{\mathrm{vi} / \text { th }}$ represents either viscous or thermal wavenumbers [20].

\subsubsection{Indicators}

In order to clarify the filter effect described by Tonndorf and its role on the $\mathrm{OE}$, a transfer function is sought between the source and the TM. According to Figure 2, the acoustic pressure at the TM is equal to that generated by the source $\mathrm{Q}$ because the EC downstream section is governed by its compressibility effect in all cases at LF. The acoustic pressure generated by the source depends, however, on the acoustic impedance seen by the source, which differs between all cases. On the other hand, the volume velocity transferred to the TM is not equal to that imposed by the source and can differ in each case. A transfer function in terms of volume velocity between the volume velocity imposed by the EC wall $q_{\text {wall }}$ and that transferred to the TM $\widehat{q}_{\mathrm{TM}}$ is computed as

$$
\widehat{T}_{\mathrm{q}}^{k}=\widehat{q}_{\mathrm{TM}}^{k} / q_{\mathrm{wall}}, \quad k \in\{\text { open, perf }- \text { occl, part }- \text { occl }\} .
$$

In equation (1), the value of $q_{\text {wall }}$ can be chosen arbitrarily since it is a transfer function whereas $\widehat{q}_{\mathrm{TM}}^{k}$ is computed using the EA model in each case and depends on all acoustic impedances and localized constants involved (see Fig. 2).

Simplified expressions of volume velocity transfer functions can be developed to help their interpretation and highlight the high-pass filter effect (see Sect. 3). To do so, the acoustic impedance at the TM is first approximated to the compressibility effect of the middle ear cavity volume of acoustic compliance $C_{\mathrm{TM}}$. This approximation is reasonable for frequencies below $500 \mathrm{~Hz}[8,21]$. Secondly, the acoustic resistance $R_{\mathrm{h}}$ associated with viscous losses in the hole is omitted. Its influence is discussed in Section 3. Applied to equation (1), these approximations lead to the 
Table 1. Calculation of the localized constants $C_{\mathrm{d}}, C_{\mathrm{u}}, L_{\mathrm{u}}, L_{\mathrm{rad}}$, $R_{\mathrm{h}}, L_{\mathrm{h}}, L_{\text {dis }}$ and $L_{\text {rad }}^{h}$ included in the EA model. $\rho_{0}=1.2 \mathrm{~kg} \mathrm{~m}^{-3}$ is the air density, $c_{0}=343 \mathrm{~m} \mathrm{~s}^{-1}$ is the sound speed and $\mu=1.8313 \times 10^{-5} \mathrm{~Pa} \mathrm{~s}$ is the air dynamic viscosity. $H(\alpha)$ represents the correction factor of the discontinuity mass $L_{\text {dis }}$ as a function of $\alpha$, the ratio of the hole and the EC entrance radius, and can be approximated by $H(\alpha) \approx 1-1.25 \alpha$ for $\alpha \ll 1$ [18].

$C_{\mathrm{d}}=\int_{0}^{l_{\mathrm{c}}} \frac{\pi r_{\mathrm{EC}}^{2}}{\rho_{0} c_{0}^{2}} \mathrm{~d} z \quad C_{\mathrm{u}}=\int_{l_{\mathrm{c}}}^{l_{\mathrm{EC}}} \frac{\pi r_{\mathrm{EC}}^{2}}{\rho_{0} c_{0}^{2}} \mathrm{~d} z \quad L_{\mathrm{u}}=\int_{l_{\mathrm{c}}}^{l_{\mathrm{EC}}} \frac{\rho_{0}}{\pi r_{\mathrm{EC}}^{2}} \mathrm{~d} z \quad L_{\mathrm{rad}}=\frac{8 \rho_{0}}{3 \pi^{2} r_{\text {ent }}}$ $R_{\mathrm{h}}=\frac{\sqrt{2 \rho_{0} \omega \mu}}{\pi r_{\mathrm{h}}^{3}} l_{h} \quad L_{\mathrm{h}}=\frac{\rho_{0} l_{\mathrm{h}}}{\pi r_{\mathrm{h}}^{2}} \quad L_{\mathrm{dis}}(\alpha)=\frac{8 \rho_{0}}{3 \pi^{2} r_{\mathrm{h}}} H(\alpha) \quad L_{\mathrm{rad}}^{\mathrm{h}}=\frac{8 \rho_{0}}{3 \pi^{2} r_{\mathrm{h}}}$

following straightforward expressions for the three cases of Figure 2:

$$
\begin{gathered}
\widehat{T}_{\mathrm{q}}^{\text {open }} \approx \frac{-\omega^{2}\left(L_{\mathrm{u}}+L_{\mathrm{rad}}\right) C_{\mathrm{TM}}}{1-\omega^{2}\left(L_{\mathrm{u}}+L_{\mathrm{rad}}\right)\left(C_{\mathrm{d}}+C_{\mathrm{TM}}\right)}, \\
\widehat{T}_{\mathrm{q}}^{\text {perf-occl }} \approx \frac{C_{\mathrm{TM}}}{C_{\mathrm{u}}+C_{\mathrm{d}}+C_{\mathrm{TM}}}, \\
\widehat{T}_{\mathrm{q}}^{\text {part-occl }} \approx \frac{-\omega^{2}\left(L_{\mathrm{u}}+L_{\mathrm{h}}^{\mathrm{eq}}\right) C_{\mathrm{TM}}}{1-\omega^{2}\left[L_{\mathrm{h}}^{\mathrm{eq}} C_{\mathrm{u}}+\left(L_{\mathrm{u}}+L_{\mathrm{h}}^{\mathrm{eq}}\right)\left(C_{\mathrm{d}}+C_{\mathrm{TM}}\right)\right]} .
\end{gathered}
$$

The OE is defined in terms of TM acoustic pressure $\widehat{p}_{\text {TM }}$ by

$$
O E_{\mathrm{m}}=20 \log _{10}\left(\left|\widehat{p}_{\mathrm{TM}}^{m} / \widehat{p}_{\mathrm{TM}}^{\text {open }}\right|\right), \quad m \in\{\text { perf }- \text { occl, part }- \text { occl }\} .
$$

Since the TM acoustic pressure is related to the associated volume velocity by $\widehat{p}_{\mathrm{TM}}^{k}=\widehat{Z}_{\mathrm{TM}} \times \widehat{q}_{\mathrm{TM}}^{k}$, the OE can be rewritten in terms of volume velocity transfer functions $\widehat{T}_{\mathrm{q}}^{k}$ such as

$$
\begin{aligned}
\mathrm{OE}_{\mathrm{m}} & =20 \log _{10}\left(\left|\widehat{T}_{\mathrm{q}}^{m} / \widehat{T}_{\mathrm{q}}^{\text {open }}\right|\right), \\
m & \in\{\text { perf }- \text { occl, part }- \text { occl }\} .
\end{aligned}
$$

Compared to equation (5), equation (6) of the $\mathrm{OE}$ is strictly limited to occlusions (perfect or partial) performed at the EC entrance such that the volume velocity imposed by the EC wall is equal to the open case. If the occlusion occurs deeper in the EC, the OE computed using equation (6) would be weighted by the ratio of volume velocity imposed by the EC wall in occluded and open cases.

\section{Results and discussions}

The moduli of the volume velocity transfer function defined by equation (1) are displayed in Figure 4 in $\mathrm{dB}$ for the open, occluded and partially occluded (hole) cases. Consider first the open case. The proportion of volume velocity transferred between the vibrating EC wall and the TM is the lowest at $100 \mathrm{~Hz}$ (minimum frequency of interest) and increases up to $1 \mathrm{kHz}$ (maximum frequency of interest). To interpret this result, equation (2) gives an

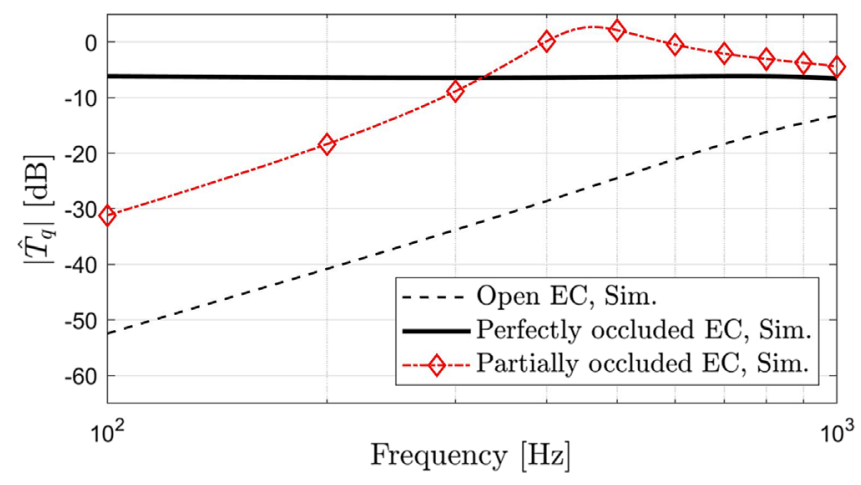

Figure 4. Level in $\mathrm{dB}$ (factor 20) of the volume velocity transfer function $\hat{T}_{\mathrm{q}}$ defined between the EC wall and the TM in open, perfectly occluded and partially occluded (with a hole) cases computed using equation (1).

approximation of the volume velocity transfer function. The latter exhibits a second order high-pass filter. As the frequency increases, the inertia effect of the EC upstream section $j \omega\left(L_{\mathrm{u}}+L_{\mathrm{rad}}\right)$ imposes more "opposition" to the volume velocity transfer, unlike the compressibility effect of the EC downstream section $\left[j \omega\left(C_{\mathrm{d}}+C_{\mathrm{TM}}\right)\right]^{-1}$, which diminishes with frequency. The conjunction of both phenomena is responsible for the increase of the volume velocity transferred between the EC wall and the TM with frequency squared (+40 dB/decade, see Fig. 4). This transfer is maximum at the quarter-wavelength acoustic resonance of the open $\mathrm{EC}$ around $3 \mathrm{kHz}$ (out of the frequency range of interest).

As suggested in introduction, the open EC high-pass filter effect is therefore not related to acoustic dissipation at the EC opening. Indeed, the acoustic resistance of radiation representing the acoustic dissipation at the EC opening can be neglected and the open EC high-pass filter effect is still there (see Eq. (2)). Therefore, the explanation of the high-pass filter effect provided by Tonndorf [7] and the interpretations of the filter effect in terms of acoustic energy transfer seen in the literature are not accurate. Indeed, the open EC high-pass filter effect is caused by the acoustic radiation of the open EC entrance, which is governed at LF by the acoustic mass of radiation $L_{\mathrm{rad}}$. In practice, acoustic radiation include dissipative effect through an acoustic resistance associated with the acoustic mass of radiation. However, as mentioned above, this acoustic resistance has little influence on the acoustic pressure radiated by the EC wall in the EC cavity and can be neglected [8]. The previous discussion clarifies and deepens the understanding of the high-pass filter effect presented by Tonndorf [5].

In the perfectly occluded case, the proportion of volume velocity transferred between the $\mathrm{EC}$ wall and the TM is approximately constant with frequency (see Fig. 4). In this case, the EC upstream section is governed by its compressibility effect rather than its inertia effect. The high-pass filter effect thus vanishes (see Eq. (3)). From $100 \mathrm{~Hz}$ to $1 \mathrm{kHz}$, the volume velocity transferred through the TM is 55 to $10 \mathrm{~dB}$ greater in the occluded case compared to the open case. Hence, the volume velocity transferred through the middle 
ear (up to the cochlea) in addition to that transferred by bone-conduction via the ligaments connecting the ossicles to the tympanic cavity (not accounted for here), is significantly increased when the EC is occluded [13]. As a result, while the outer ear pathway is of little influence on the hearing by bone-conduction when the EC is open [14, 22], it becomes predominant when the EC is occluded $[13,14]$.

When the EC is partially occluded, Figure 4 shows that the volume velocity transferred between the $\mathrm{EC}$ wall and the TM increases with frequency by approximately $+40 \mathrm{~dB} /$ decade (similar to the open case) until it reaches a maximum around $450 \mathrm{~Hz}$. This transfer is then almost constant with frequency (similar to the occluded case). The approximation given by equation (4) exhibits a second order high-pass filter, just like the open EC. The partially occluded EC, however, behaves as a Helmholtz resonator rather than a quarter-wavelength resonator. The Helmholtz resonance frequency is given by $f_{0}=\left[4 \pi^{2}\left(L_{\mathrm{h}}^{\mathrm{eq}} C_{\mathrm{u}}+\left(L_{\mathrm{u}}+L_{\mathrm{h}}^{\mathrm{eq}}\right) \times\right.\right.$ $\left.\left.\left(C_{\mathrm{TM}}+C_{\mathrm{d}}\right)\right)\right]^{-1 / 2}$. This resonance is mainly damped by the acoustic resistance of the TM and to a lesser extent on the hole viscous resistance $R_{h}$, depending on the hole radius. These resistances are neglected in the approximation given by equation (4) which is not used in Figure 4 for this reason. If the acoustic mass $L_{\mathrm{h}}^{\mathrm{eq}}$ of the hole is significantly higher than that of the EC upstream section $L_{\mathrm{u}}$ (hole radius small enough or length long enough), the hole corresponds to the neck of the Helmholtz resonator whereas the whole EC acts as the resonator cavity. On the contrary, if the length of the hole tends to zero and if its radius goes to that of the EC entrance, the partially occluded case tends to the open case (i.e., Eq. (4) tends to Eq. (2)).

The change in volume velocity transfer function between open and partially occluded cases is now investigated regarding the OE. Figure 5 displays the OE induced by the perfect and the partial occlusions of the EC entrance. In the perfect occlusion, the $\mathrm{OE}$ is maximum at $100 \mathrm{~Hz}$ and decreases with frequency by approximately $-40 \mathrm{~dB} /$ decade. This slope is similar to literature data $[3,12,13]$ and results from the removal of the open EC high-pass filter effect, as proposed by Tonndorf [6]. Tonndorf's model therefore included the minimum features to simulate the objective OE for a perfect occlusion. When the EC is partially occluded, the OE increases from $100 \mathrm{~Hz}$ to around $450 \mathrm{~Hz}$ and decreases above this frequency (see Fig. 5).

Figure 5 also displays the OE measured by Hansen [16] on a human subject for a shallow partial occlusion. Simulation and experimental data exhibit similar slopes. The model approximately predicts the resonance frequency observed in experimental data around $450 \mathrm{~Hz}$. However, the model overestimates the measured $\mathrm{OE}$ by approximately $6 \mathrm{~dB}$ in the whole frequency range of interest $(100 \mathrm{~Hz}-1 \mathrm{kHz})$. This difference in $\mathrm{OE}$ amplitude most likely comes from the difference in insertion depth between the current model (defined at the EC entrance) and the measurement (shallow insertion). Indeed, since the OE decreases with increasing insertion depth, the simulated $\mathrm{OE}$ is therefore higher than the measured OE. The influence of the insertion depth on the $\mathrm{OE}$ can be accounted for by adjusting the volume velocity imposed to the occluded EC cavity $[3,13,15]$. When

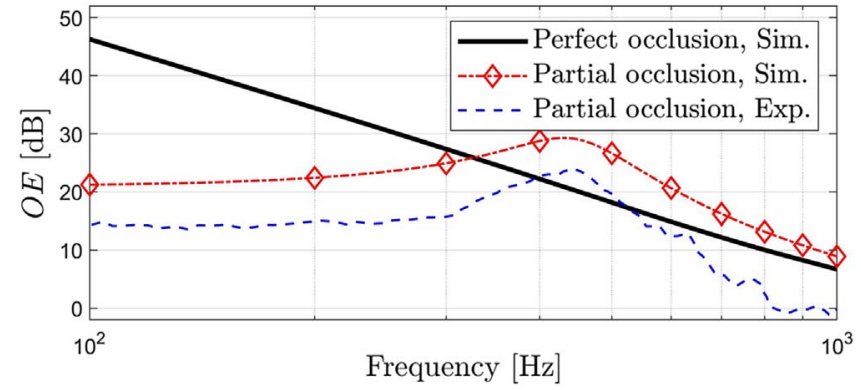

Figure 5. OE induced by a perfect and a partial (with a hole) occlusions (at the EC entrance) computed using equation (5) of the EA model. In addition, experimental data provided by Hansen [16] are also displayed for a shallow partial occlusion.

the occlusion device is deeply inserted, the $\mathrm{OE}$ is significantly reduced [1,3].

The maximum of the $\mathrm{OE}$ induced by the partial occlusion occurs at the resonance frequency (around $450 \mathrm{~Hz}$ in Fig. 5) of the Helmholtz resonator formed by the hole and the EC cavity $[15,16,23]$. Below the Helmholtz resonance, the $\mathrm{OE}$ is significantly reduced compared to the perfect occlusion and approximately constant with frequency. The amplitude of the $\mathrm{OE}$ is given by the ratio between the acoustic mass of the hole and that of the open EC. The hole acts as a low acoustic impedance pathway for the volume velocity transfer. This phenomenon has inspired the use of vent in hearing aids to decrease the $\mathrm{OE}[2,24,25]$. It is however associated with acoustic feedback which decreases the intelligibility provided by hearing aids. In the case of earplugs, an incomplete seal is generally avoided since it decreases their sound attenuation [26]. Fit testing methods have been developed to prevent this. In the simplest case, the perception of the OE can even be used as an estimate of the seal quality: the greater the perceived $\mathrm{OE}$, the greater the seal [1].

\section{Conclusion}

This paper has revisited and deepened the high-pass filter effect mentioned by Tonndorf and how this filter is changed in occluded case (perfect or partial). The EA model proposed by Tonndorf has been clarified, and used to highlight a second order high-pass filter for the volume velocity transferred between the EC wall and the TM in the open EC. The open EC high-pass filter effect is caused by the acoustic radiation of the EC opening which is governed at LF by the acoustic mass of radiation whereas acoustic dissipation at the EC opening plays a negligible role and cannot explain the high-pass filter effect. The perfect occlusion replaces the open EC high-pass filter effect by a filter constant with frequency. On the contrary, the partial occlusion (hole) exhibits a high-pass filter effect just like the open EC but behaves as a Helmholtz resonator. For a perfect occlusion, the $\mathrm{OE}$ can be described as the removal of the open EC high-pass filter effect at LF. In consequence, the volume velocity transferred from the EC cavity in the 
middle ear through the TM drastically increases, which explains the predominance of the occluded outer ear pathway on the hearing by bone-conduction at LF. For a partial occlusion, the OE results from a change in properties of the open EC high-pass filter effect, which is not removed.

\section{Acknowledgments}

The authors acknowledge the support of the Natural Sciences and Engineering Research Council of Canada (NSERC) (funding reference number RGPIN-201606795). Also, all reviewers are gratefully thanked for their critical and wise comments.

\section{References}

1.E.H. Berger: Hearing protection devices. The noise manual, Revised Fifth edition, AIHA Press, Fairfax, VA, 2003.

2. H. Mueller, K. Bright, J. Northern: Studies of the hearing aid occlusion effect. Seminars in Hearing 17, 1 (1996) 21-31.

3. S. Stenfelt, S. Reinfeldt: A model of the occlusion effect with bone-conducted stimulation. International Journal of Audiology 46, 10 (2007) 595-608. https://doi.org/10.1080/ 14992020701545880 .

4. E.H. Huizing: Bone conduction-the influence of the middle ear. Acta Oto-Laryngologica Suppl. 155 (1960) 1-99.

5. J. Tonndorf: Bone conduction. Studies in experimental animals. Acta Oto-Laryngologica Suppl. 213 (1966) 1-132.

6. J. Tonndorf: Animal experiments in bone conduction: clinical conclusions. Transactions of the American Laryngological, Rhinological and Otological Society 52 (1964) 22-41. https://doi.org/10.1177/000348946407300308.

7. J. Tonndorf: Bone conduction, in: J.V. Tobias (Ed.), Foundations of Modern Auditory Theory, Vol. 2, Academic Press, New York, 1972, pp. 195-237.

8. K. Carillo, O. Doutres, F. Sgard: Theoretical investigation of the low frequency fundamental mechanism of the objective occlusion effect induced by bone-conducted stimulation. Journal of the Acoustical Society of America 147, 5 (2020) 3476-3489. https://doi.org/10.1121/10.0001237.

9. F.K. Kuk: Perceptual consequence of vents in hearing aids. British Journal of Audiology 25, 3 (1991) 163-169.

10. M.A. Fagelson, F.N. Martin: The occlusion effect and ear canal sound pressure level. American Journal of Audiology 7, 2 (1998) 50-54.

11. S.A. Small, N. Hu: Maturation of the occlusion effect: A bone conduction auditory steady state response study in infants and adults with normal hearing. Ear and Hearing 32, 6 (2011) 708-719.

12. M. Brummund, F. Sgard, Y. Petit, F. Laville: Threedimensional finite element modeling of the human external ear: Simulation study of the bone conduction occlusion effect. Journal of the Acoustical Society of America 135, 3 (2014) 1433-1444.

13. J. Schroeter, C. Poesselt: The use of acoustical test fixtures for the measurement of hearing protector attenuation. Part II: Modeling the external ear, simulating bone conduction, and comparing test fixture and real-ear data. Journal of the Acoustical Society of America 80, 2 (1986) 505-527.

14. S. Stenfelt, T. Wild, N. Hato, R.L. Goode: Factors contributing to bone conduction: The outer ear. Journal of the Acoustical Society of America 113, 2 (2003) 902-913. https://doi.org/10.1121/1.1534606.

15. T. Zurbrügg, A. Stirnemannn, M. Kuster, H. Lissek: Investigations on the physical factors influencing the ear canal occlusion effect caused by hearing aids. Acta Acustica united with Acustica 100, 3 (2014) 527-536. https://doi.org/ 10.3813/AAA.918732.

16. M.O. Hansen: Occlusion effects - Part II - A study of the occlusion effect mechanism and the influence of the Earmould properties, Ph.D. Thesis. Department of Acoustic Technology, Technical University of Denmark, Denmark, 1998.

17. E.A.G. Shaw, M.R. Stinson: Network concepts and energy flow in the human middle ear. Journal of the Acoustical Society of America 69, 1 (1981) 43.

18. F.P. Mechel (Ed.): Formulas of acoustics. 2nd ed., Springer, Berlin; New York, 2008.

19.F.C. Karal: The analogous acoustical impedance for discontinuities and constrictions of circular cross section. Journal of the Acoustical Society of America 25, 2 (1953) 327-334.

20. M. Bruneau: Fundamentals of acoustics. John Wiley \& Sons, New York, 2013.

21. C.E. Stepp, S.E. Voss: Acoustics of the human middle-ear air space. Journal of the Acoustical Society of America 118, 2 (2005) 861-871. https://doi.org/10.1121/1.1494445.

22. S. Stenfelt: Model predictions for bone conduction perception in the human. Hearing Research 340 (2016) 135-143. https://doi.org/10.1016/j.heares.2015.10.014.

23. M. Brummund: Study of the occlusion effect induced by an earplug: Numerical modelling and experimental validation, PhD Thesis. École de technologie supérieure, Montréal, Québec, Canada, 2014.

24. R. Carle, S. Laugesen, C. Nielsen: Observations on the relations among occlusion effect, compliance, and vent size. Journal of the American Academy of Audiology 13, 1 (2002) 13 .

25. A. Winkler, M. Latzel, I. Holube: Open versus closed hearingaid fittings: A literature review of both fitting approaches. Trends in Hearing 20 (2016) 2331216516631741. https://doi. org $/ 10.1177 / 2331216516631741$.

26. G. Viallet, F. Sgard, F. Laville, H. Nélisse: Investigation of the variability in earplugs sound attenuation measurements using a finite element model. Applied Acoustics 89 (2015) 333-344. https://doi.org/10.1016/j.apacoust.2014.10.007. 